\title{
AN ANALYSIS OF GAPPING \\ IN A METAGRAMMATICAL ORGANIZATION OF GRAMMAR*
}

\author{
Toshiyuki Tonolke
}

Tokyo Metropolitan University

\begin{abstract}
The aim of this paper is to propose an analysis of Gapping in a metagrammatical organization of grammar. A strategy for defining a grammar of a natural language by metagrammatical mechanisms is adopted in the framework, now called Generalized Phrase Structure Grammar, a generative grammar developed in such works as Gazdar 1981, 1982, Gazdar and Pullum 1981, 1982, Gazdar, Pullum, and Sag 1981, and Gazdar, Klein, Pullum, and Sag 1982, in press. The analysis of Gapping given below is along the general lines of Generalized Phrase Structure GRAmmar. The rules necessary for generating gapped conjuncts are defined in dependence on the existence of rules for the corresponding nongapped conjuncts. The first half of the paper summarizes the discussion on coordination and Gapping by Sag, Gazdar, Wasow, and Weisler 1984 and the second half proposes some rules and conditions which insure generation of various Gapping sentences.
\end{abstract}

\section{Introduction}

Generalized Phrase Structure Grammar (henceforth, GPSG) is a grammatical framework in which syntax consists only of context-free phrase structure rules and operations such as transformations are not assumed. In GPSG there is only one level of syntactic representation; logical forms or f-structures as syntactic representation are not postulated. Another important characteristic of GPSG is that following Montague 1974, semantics consists of semantic translations and that each semantic translation is defined to have a one-to-one correspondence to a syntactic rule. ${ }^{1}$

* I am very grateful to Takao Gunji, Yasunari Harada, and Heizo Nakajima for their suggestions and encouragement, though they do not necessarily approve of the proposals given in the paper. I am also grateful to the members of Linguistic Circle of Tokyo Metropolitan University.

1 One point of clarification is in order. In the following discussion I shall not attempt to compare GPSG with other frameworks of generative grammar. For motivations for restricting to context-free phrase structure rules, see, e.g. Gazdar 
The most innovative characteristic of GPSG is its metagrammatical ORGANIZATION OF GRAMMAR. In GPSG a grammar of a natural language is generated as shown in Figure 1.2

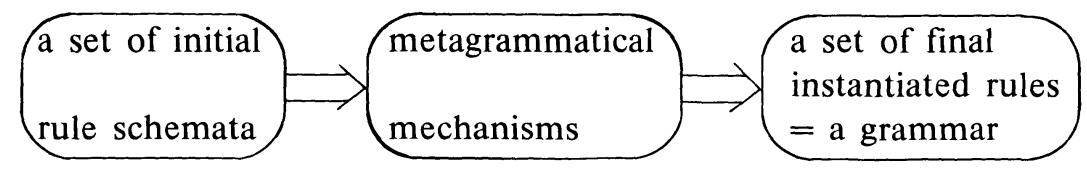

The organization of grammar in GPSG

Figure 1.

Sag, Gazdar, Wasow, and Weisler (1984: 11, henceforth, SGWW) characterize GPSG as just a set of ID [Immediate Dominance] rules and metarules taken together with a lexicon and a set of ancillary grammatical principles.' According to SGWW's formulation the theory of GPSG contains six components:

(1) i) Immediate Dominance (ID) Rules

a. Lexical ID Rules

b. Nonlexical ID Rules

ii) Metarules

iii) Lexicon

iv) Feature Instantiation Principles

v) Linear Precedence Statements

vi) Semantic Interpretation Principles

Diagrammatically, SGWW's characterization of GPSG can be shown as follows: ${ }^{3}$

1982.

Details of the framework of GPSG will not be given. See, e.g. Gunji 1983 and Gazdar, Klein, Pullum, and Sag (in press: henceforth, GKPS).

${ }^{2}$ Figure 1 is due to Gunji (in preparation, draft 1.0: Chapter 1, adapted).

3 Familiar context-free phrase structure rules define two kinds of relations, i.e. immediate dominance and linear precedence relations. For arguments for separation of the relations, see Gazdar and Pullum 1982.

The organization of grammar in GPSG given by Gazdar and Pullum 1982 does not distinguish Lexical ID Rules from Nonlexical ID Rules. Flickinger 1983 proposes that input to metarules should be restricted to Lexical ID Rules. The organization of grammar given in Figure 2 is a modification of Gazdar and Pullum 1982 by Flickinger 1983. 


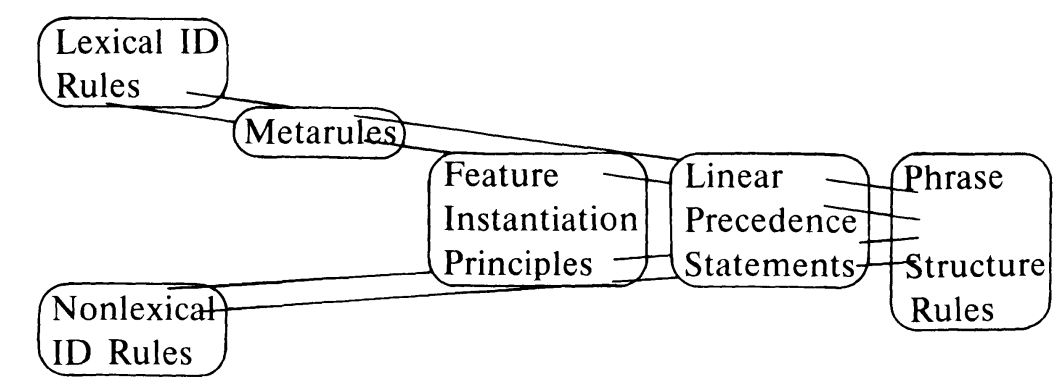

The organization of grammar in the recent version of GPSG Figure 2.

The discussion given below will follow mainly SGWW's formulation shown in Figure 2.

\section{Preliminaries}

1.1. Syntactic Categories and Syntactic Features. In the earlier version of transformational grammar (henceforth, TG), categories were first thought to be undecomposable into anything. But Chomsky 1970 proposed that syntactic categories can be taken to be bundles of abstract syntactic features. This idea has been utilized extensively in GPSG.

In the theory of features we are assuming here, if some feature is postulated, its possible values must be stated. For example, we assume the syntactic features in 2 , where $\mathrm{f}$ stands for a feature name and $\mathrm{S}(\mathrm{v})$ stands for a set of possible values for the feature:

(2) $\mathrm{f}$

$$
\begin{array}{cc}
\mathrm{f} & \mathrm{S}(\mathrm{v}) \\
\text { BAR } & \{0,1,2\}^{4} \\
\mathrm{~N} & \{+,-\} \\
\mathrm{V} & \{+,-\}
\end{array}
$$

3 illustrates some possible categories:

(3) a. $\{<\mathrm{N},->,\langle\mathrm{V},+\rangle,<\mathrm{BAR}, 0\rangle\}$ : $\mathrm{V}^{0}$

b. $\quad\{<\mathrm{N},+>,<\mathrm{V}, \rightarrow>,<\mathrm{BAR}, 2>\}: \mathrm{N}^{2}$

One of the most important assumptions made by SGWW is the abandonment of the following rather common stipulation.

(4) Only a fully specified category may label a node. (p. 4)

${ }^{4}$ In the current version of GPSG, a two-bar version of X-bar theory is adopted. See Gazdar, Klein and Pullum, eds. (1983: Chapter 1) for arguments of X-bar theory by proponents of GPSG. 
The absence of the stipulation of 4 is crucial to the analysis of coordination given by SGWW.

1.2. The Distribution of Conjunctions. In the version of GPSG we are assuming here, there is a feature $\mathrm{CONJ}$ (=conjunction), whose values are enumerated in the following set:

( 5 ) \{and, both, but, NIL, either, neither, nor, or $\}$ SGWW give the following rule to expand categories which include CONJ in their domain:

( 6 ) $\mathrm{X}[\mathrm{CONJ} \alpha] \rightarrow \alpha, \mathrm{H}$

where $\alpha \in\{$ and, both, but, either, neither, nor, or $\}$ and $\mathrm{X}$

stands for an archicategory and $\mathrm{H}$ stands for the head of $\mathrm{X}$.

SGWW assume two kinds of coordination: one which has only two conjuncts, and the other which has an unlimited number of conjuncts. Accordingly, SGWW postulate two rule schemata: one for the two-conjunct coordination, and the other for unlimited-numberconjunct coordination.

(7) Binary Coordination Schema (BCS)

a. $\mathrm{X} \rightarrow \mathrm{H}\left[\mathrm{CONJ} \alpha_{0}\right], \mathrm{H}\left[\mathrm{CONJ} \alpha_{1}\right]$

b. $\quad \alpha$ is in $\langle$ both, and $\rangle$, <either, or $\rangle,\langle$ NIL, but $\rangle$.

(8) Iterative Coordination Schema (ICS) ${ }^{5}$

a. $\mathrm{X} \rightarrow \mathrm{H}\left[\mathrm{CONJ} \alpha_{0}\right], \mathrm{H}\left[\mathrm{CONJ} \alpha_{1}\right]^{+}$

b. $\alpha$ is in $\langle$ and, NIL $\rangle,\langle\mathrm{NIL}$, and $\rangle,<$ neither, nor $\rangle,<$, $<$, NIL $>,\langle$ NIL, or $>$.

SGWW propose the following LP statements in a collapsed form:

(9) $\mathrm{CONJ} \alpha_{0}>\mathrm{CONJ} \alpha_{1}$

where $\alpha_{0} \in\{$ both, NIL, either, neither $\}$ and $\alpha_{1} \in\{$ and, but, nor, or $\}$

1.3. A Unitary Notion of Coordination. SGWW observe that 'transformational grammar has never been able to capture a unitary notion of coordination, for reasons that were fundamental to the nature of the theory.' (p. 15). They consider the following sentences:

(10) a. Kim sang and Sandy danced.

b. Kim and Sandy met.

c. Kim sang and was accompanied by Sandy.

${ }^{5}+$ is an abbreviatory notation. For example, $\mathrm{A}^{+}$means that $\mathrm{A}$ is allowed to appear any positive times more than 0 .

$\mathrm{A}^{+}=\{\mathrm{A}, \mathrm{AA}, \mathrm{AAA}, \ldots\}$ 
Sentences like 10a, 10b are generated directly by base rules. But 10c, which is also an example of coordination, is derived in a completely different fashion in the standard version of TG. Any version of TG in which passive sentences are derived by transformation cannot capture the property shared by all the sentences in 10 . In Section 4 we will consider the question of whether or not behavior of Gapping is captured by the UNITARY NOTION OF COORDINATION.

1.4. Features in Coordination. It is often assumed that in coordinate constructions the mother node of conjuncts bears the same syntactic category as its daughters. SGWW cite the following sentences as examples to show that this is not always the case:

(11) a. We walked slowly and with great care. (Adv and PP)

b. Terry turned out to be longwinded and a bully. (AP and NP)

(12) a. *The longwinded and a bully man was my brother.

b. *Soon longwinded and a bully man started shouting again. Two things call for an explanation.

i) what is the category of the mother node in $11 \mathrm{a}$ and $11 \mathrm{~b}$ ?

ii) what makes the sentences in 12 ungrammatical?

SGWW propose to introduce a feature PRD (predicative) and assume the following ID rules for the verb be:

(13) $\mathrm{V}^{1} \rightarrow \mathrm{H}^{0}, \mathrm{X}^{2}$ [PRD]

$\mathrm{X}^{2}$ is an ARCHICATEgory which stands for $\{<\mathrm{BAR}, 2>\}$. In other words, $\mathrm{X}^{2}$ is a category which is specified only for the syntactic feature BAR, and is unspecified for other features. If an archicategory like $\mathrm{X}^{2}$ is to be excluded from the grammar, then the following set of rules is needed for the predicative verb be in the grammar:

(14) a. $\mathrm{V}^{1} \rightarrow \mathrm{H}^{0}, \mathrm{~N}^{2}[\mathrm{PRD}]$

b. $\mathrm{V}^{1} \rightarrow \mathrm{H}^{0}, \mathrm{~A}^{2}[\mathrm{PRD}]$

c. $\mathrm{V}^{1} \rightarrow \mathrm{H}^{0}, \mathrm{P}^{2}[\mathrm{RPD}]$

d. $\mathrm{V}^{1} \rightarrow \mathrm{H}^{0}, \mathrm{~V}^{2}[\mathrm{PRD}]$

The rule in 13 is much simpler than those in 14 and it takes care of all the following sentences:

(15) a. Kim was a banker.

b. Dana was quite competent.

c. Leslie was in the flood zone.

d. Ronnie was talking to Lou.

e. Jean was given a prize. 
Furthermore, SGWW propose a similar treatment for the verbs become and consider.

(16) a. Pat has become a republican. $(\mathrm{RRD},+\mathrm{N})$

b. Gerry became quite conservative.

c. ${ }^{*}$ Connie has become of the opinion that we should get out.

d. *Tracy became awarded a prize.

e. ${ }^{*}$ Chris will become talking to colleagues.

(17) a. We considered $\mathrm{Mel}$ a friend. (RRD, $-\mathrm{PRP}=$ present participle)

b. They had considered them quite conservative.

c. Ronnie considers them in need of our help.

d. *Jan will consider them talking to too many outsiders.

1.5. The Head Feature Convention. One of the feature instantiation principles is called Head Feature Convention (henceforth, HFC).

(18) The Head Feature Convention (HFC) ${ }^{6}$

The HEAD of a head must be instantiated as an extension of the HEAD of the mother.

The HFC allows the distribution of features shown in 19: the intersection of the HEAD feature specification of head daughters, i.e. $\mathrm{N}^{2}$ [PRD] and $\mathrm{A}^{2}$ [PRD], is equivalent to the HEAD feature specification of their mother, namely, [PRD, $+\mathrm{N}$ ].

(19)

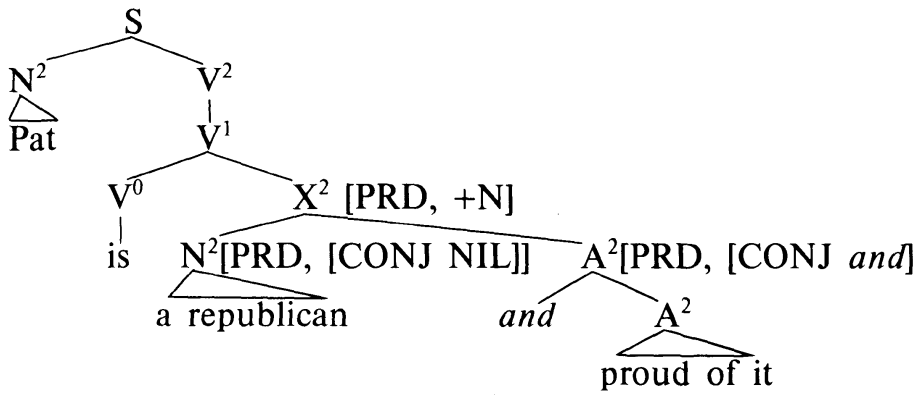

SGWW account for 11a by assuming that $\mathrm{Adv}^{2}$ slowly and $\mathrm{P}^{2}$ with great care share an adverbial feature [MANNER].

SGWW suggest that a similar analysis is also possible for other adverbials and give examples of feature TEMP (temporal):

${ }^{6}$ Several versions of the HFC have been proposed in the literature. The version given in 18 is the one revised by Farkas et al. 1983. 
(20) They wanted to leave tomorrow or on Tuesday. ( $\mathrm{Adv}^{2}$ [TEMP], $\mathrm{P}^{2}[$ TEMP])

The ungrammaticality of 12 is explained by SGWW by disallowing archicategories in prenominal adjective phrases.

2. GAPPING

2.1. Fundamental Properties of Gapping. Gapping is restricted to coordinate constructions and cannot take place in other types of sentences such as 21:

(21) *Sandy played the guitar while (after, before, since, although ...) Betsy the recorder.

Any treatment of Gapping must account for the following two fundamental properties observed in Gapping sentences. In Gapping the verb in the nongapped conjunct must be identical to the verb in the gapped conjunct, and furthermore, they share the same value for the feature TENSE.

(22) a. Alice ordered pork chops and Ben $\phi x d e r d \phi$ a Coke.

b. *Alice ordered pork chops and Ben $\phi t a k k$ a Coke.

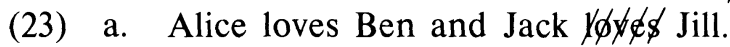

b. *Alice loves Ben and Jack $\not \phi \Varangle ф \emptyset$ Jill.

2.2. Sag, Gazdar, Wasow, and Weisler's (1984) Proposal for GapPING. Consider the following sentences:

(24) Kim likes Sandy, and Lee Leslie.

(25) Pat wanted to try to go to Berne, and Chris

$$
\left\{\begin{array}{l}
\text { to try to go to Rome. } \\
\text { to go to Rome. } \\
\text { to Rome. }
\end{array}\right\}
$$

(26) Kim gave a dollar to Bobbie and a dime to Jean.

(27) Kim went to the store, and (then) Lou.

SGWW propose 'a single ellipsis principle' (p. 33) on which sentences like 24-27 are accounted for. SGWW observe that all the sentences 24-27 share some common properties like the following:?

7 SGWW propose to treat Stripping in the same manner as Gapping, since stripping shares some properties with Gapping. The interpretation of 27 relevant to our discussion is Kim went to the store, and (then) Lou went to the store. One of my informants said that if the item then is omitted, the resulting sentence is nearly ungrammatical. Further, one can imagine another interpretation of 27 . It is Kim 
i) 'there is a non-initial conjoined element that contains a sequence of phrasal elements which is not generated freely as such elsewhere in the grammar.'

ii) 'there is a dependency between the form of these remnants and the presence of appropriate elements in the appropriate positions in the preceding conjoined clause.'

SGWW give the following sentences which are ungrammatical because of the absence of the properties given above.

(29) a. *Kim likes Sandy, and Lee to Leslie.

b. *Pat wanted to go to Berne, and Chris going to Rome.

c. ${ }^{*}$ Kim gave a dollar to Bobbie and a dime into his pocket.

d. *Kim likes Lee, and to Ronnie.

Consider the following sentences:

(30) a. Leslie is rather foolish, and Lou a complete idiot.

b. Kim seems to be just surviving, and Terry in dire need of our help.

c. We consider Leslie rather foolish, and Lou a complete idiot.

(31) a. Pat has become crazy, and Chris an incredible bore.

b. Pat has become crazy, and Chris depressed.

c. *Pat has become crazy, and Chris in good spirit.

These sentences are correctly accounted for by assuming appropriate syntactic features and ID rules for verbs be, seem, consider, become, etc., as we have seen in Section 1.4.

went to the store, and (then) Kim went to Lou. This interpretation is awkward. But one can interpret 27 , if in isolation, in either way. Stripping sentences force hearers to substitute much more elements than Gapping sentences. This difference suggests that Stripping is much more dependent on context than Gapping.

Moreover, some items like then, as in 27, are often added to Stripping sentences and the resulting sentences are more acceptable. Jackendoff (1971: 26) cites the following sentences (judgments are his):

i) *Bill ate the peaches, and Harry.

ii) ?Bill ate the peaches, and Harry ate the peaches.

iii) Bill ate the peaches, and Harry, too.

Notice that ii is prefixed a question mark. Items like then (as in the sentence 27) and too (as in iii) seem to be of metalanguage in nature. Such properties of Stripping suggest that it needs a separate treatment. (See Kaplan 1984 for a detailed examination of the properties of too. See Pullum and Gazdar 1982 for a discussion of the property of respectively.) 
SGWW conclude their examination of Gapping as follows:

Gapping is a discourse anaphoric process, not a rule of sentence grammar, despite claims to the contrary made by Williams (1977) and Neijt (1979). The rules of sentence grammar thus have only to generate structures consisting of a conjunction followed by any number of $\mathrm{X}^{2}$ 'remnants' and a principle must be stated to predict the interpretation of the sequence of remnant from the preceding, directly juxtaposed linguistic context. (p. 36)

And they propose a schematic ID rule like:

(32) $\mathrm{V}^{2}[\mathrm{ELL},[\mathrm{CONJ} \alpha]] \rightarrow \alpha, \mathrm{X}^{2}[\mathrm{FOC}]^{+}$ $(\mathrm{ELL}=\text { elliptical, } \mathrm{FOC}=\text { focal })^{8}$

The elliptical structures in question are distinguished by the feature ELL. Further, ellipsis remnants are generally assigned a contrastive stress, and this property is reflected by the feature FOC. Note that in the framework we are assuming, S's are distinguished from VP's by the feature SUBJ. S's are VP's with a feature specification $\langle$ SUBJ, $+>$. Thus, $\mathrm{V}^{2}$ in 32 stands for both an $\mathrm{S}$ and a VP.

SGWW give the following examples as structures resulting from the rule in 32 (p. 37):

(33)

a.

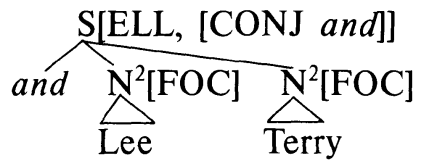

(34) b. $\mathrm{V}^{2}[\mathrm{ELL},[\mathrm{CONJ}$ but $]]$

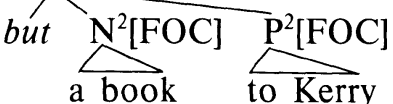

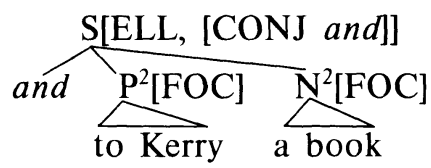

SGWW assume that gapped structures like 33-34 are interpreted 'by substituting the remnants for the appropriate corresponding elements in a preceding directly juxtaposed structure.' (p. 37). And if the structure resulting from the substitution is a structure which cannot be generated by the grammar independently, the gapped structure $\mathrm{V}^{2}[$ ELL] will obtain no interpretation.

SGWW exemplify their analysis of ellipsis constructions as shown below:

\footnotetext{
${ }^{8}$ Ellipsis in subordinate clauses is not in the domain of feature ELL.
} 
(35) The Case of S-level Gapping

a. Terry likes Stacy and Tracy Lee.

b.

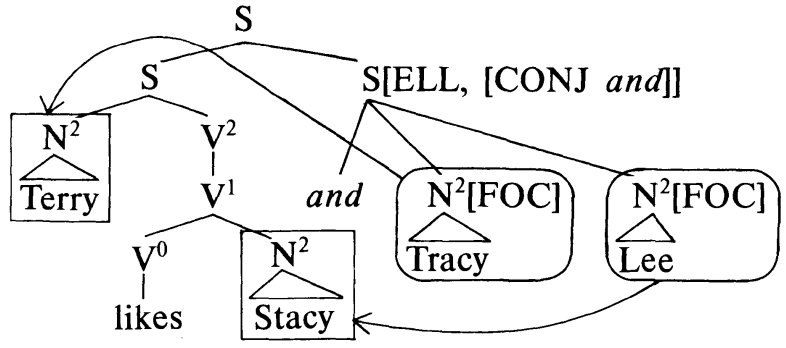

The semantic interpretation for the sentence in $35 \mathrm{a}$ is given 'by interpreting the result of substituting the circled elements for the boxed elements in fashion illustrated.' (p. 38).

(36) The Case of VP-Level Gapping

a. Sandy gave the book to Lee and the record to Kim.

b.

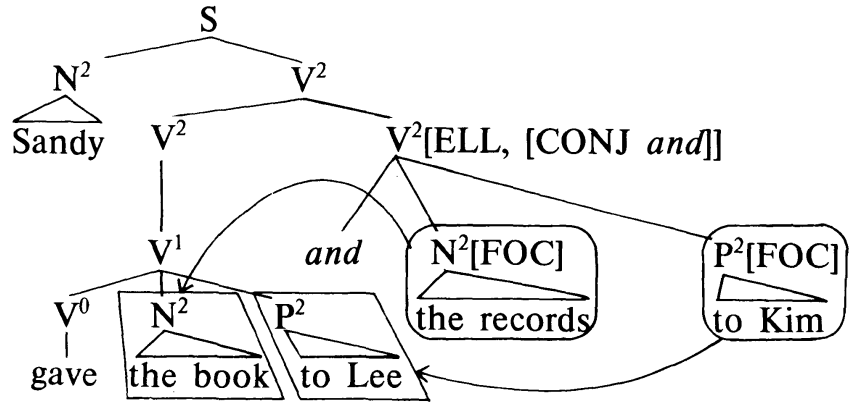

The semantic interpretation for the sentence in 36a is given in the same way as the case of $35 \mathrm{a}$. As the resulting interpretations, SGWW give the following:

a. and'(like'(Stacy*)(Terry*), like'(Lee*)(Tracy*))

b. and'(give'(Lee*) (the'(book')), give'(Kim*) (the'(records')) (Sandy*)

SGWW's concluding remarks on their analysis of Gapping can be summarized as follows (p. 39):

(38) SGWW'S Concluding Remarks

i) Gapping (with two or more than two remnants) and coordinate instances of StRIPPING (with only one remnant) can be accounted for in a unified way.

ii) SGWW's analysis allows all the examples in 24-27 to be generated, but not the examples in 29 
iii) the sentences in 30 are allowable as, for example, a substitution of $\mathrm{N}^{2}$ for $\mathrm{A}^{2}$, since the verb be is introduced by a rule like $\mathrm{V}^{1} \rightarrow \mathrm{H}^{0}, \mathrm{X}^{2}$ [PRD].

iv) although many further factors may be involved in Gapping (they may be extra-grammatical in nature), SGWW's proposal for the syntactic substitution operation presented above, is a plausible candidate for the grammatical part of explanation of Gapping.

Although their general observations on Gapping are quite reasonable, the status of their substitution operation is not clear and some elaborations are needed to capture fundamental properties of Gapping in the organization of grammar.

\section{A More Explicit Formulation of Gapping}

First, there is no transformation (movement or deletion) in GPSG, and therefore, in principle, all the necessary ID rules for a given language have to be specified either as separate stipulation or through metagrammatical mechanisms. Needless to say, if some of them can be collapsed into a single rule or a rule schema, or can be defined in an inductive way, a better move toward a genuine generalization of the language is attained.

In the subsequent sections we will make an attempt to define rules for gapped conjuncts in capturing the fundamental properties of Gapping discussed above.

3.1. Cases Which Can Be Analyzed with Subcategorization Frames. ${ }^{9}$ The minimum task for an analysis of Gapping is to guarantee the generation of simple Gapping sentences like 24 and 26. For the case of S-level Gapping $\left(V^{2},+\right.$ SUBJ) like 24 , the following ID rules are needed: $:^{10}$

${ }^{9}$ A terminological clarification may be in order. $G_{A P P I N G}$ is used to refer to a phenomenon. Gapping sentences are examples of Gapping. Nongapped conjuncts is used to refer to the initial conjunct of Gapping sentences. For example, the conjunct Kim likes Sandy of the sentence in 24 is a nongapped conjunct and the conjunct Lee Leslie is a Gapped conjunct.

${ }^{10}$ Subcategorization frames for verbs are distinguished by distinct numbers assigned to them.

Auxiliary verbs are not treated in this paper. The grammaticality difference between the following strings are not considered. 
(39) a. $S \rightarrow$ S[TNS t], S[ELL, [TNS t, [CONJ and]]]

b. $\quad S[T N S t] \rightarrow N^{2}, V^{2}[T N S t]$

c. $\mathrm{V}^{2}[$ TNS $\mathrm{t}] \rightarrow \mathrm{V}^{1}[$ TNS $\mathrm{t}]$

d. $\quad \mathrm{V}^{1}[$ TNS $\mathrm{t}] \rightarrow \mathrm{V}^{0}$ [TNS $\left.\mathrm{t},[1]\right], \mathrm{N}^{2}$ (V[1]: like, kill, ...)

e. S[ELL, [TNS t, [CONJ and]]] $\rightarrow$ and, $\mathrm{N}^{2}[\mathrm{FOC}], \mathrm{N}^{2}[\mathrm{FOC}]$

39 guarantees that sentence 24 is admitted by the grammar. They are, of course, not general enough to admit many other Gapping sentences.

Next, we need the rules in 40 to generate VP-Level $G_{A P P I N G}\left(V^{2}\right.$, -SUBJ) sentences like 26.

(40) a. $\mathrm{S} \rightarrow \mathrm{N}^{2}, \mathrm{~V}^{2}$

b. $\mathrm{V}^{2} \rightarrow \mathrm{V}^{2}[\mathrm{TNS} \mathrm{t}], \mathrm{V}^{2}[\mathrm{ELL}$, [TNS $\mathrm{t}$, [CONJ and]]]

c. $V^{2}[$ TNS $t] \rightarrow V^{1}$ [TNS t, [2]]

d. $\mathrm{V}^{1}[\mathrm{TNS} \mathrm{t},[2]] \rightarrow \mathrm{V}^{0}$ [TNS $\left.\mathrm{t},[2]\right], \mathrm{N}^{2}, \mathrm{P}^{2}[$ to] (V[2]: give, hand, ...)

e. $\mathrm{V}^{2}[\mathrm{ELL},[\mathrm{TNS} \mathrm{t},[\mathrm{CONJ}$ and $]]] \rightarrow$ and, $\mathrm{N}^{2}[\mathrm{FOC}], \mathrm{P}^{2}[$ to, [FOC]]

Other verbs involved in VP-Level GAPPING, if they are treated with their frame numbers, do not cause serious problems. We will restrict our attention to S-LEVEL GAPPING hereafter.

We assume that simple gapped S's can be defined in the grammar depending on the existence of the corresponding simple nongapped S's. Consider structure 41:

(41)

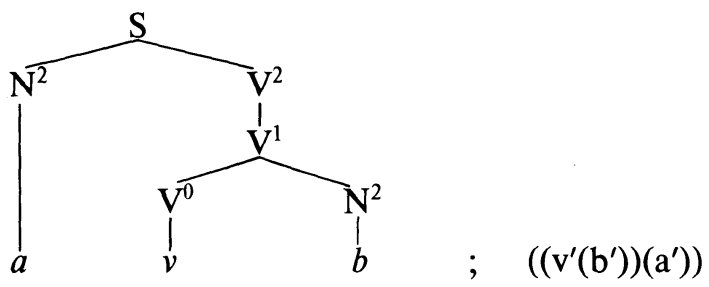

(Irrelevant details are omitted. Small letters stand for lexical items.)

Structure 41 is responsible for sentences like 42 :

John may have been examined by a doctor, and Ed

i) have been being inspected by a policeman.

ii) been being inspected by a policeman.

iii) being inspected by a policeman.

iv) inspected by a policeman.

v) by a policeman. 
(42) Kim likes Sandy.

The ID rule expanding $\mathrm{V}^{1}$ in 41 is:

(43) $\mathrm{V}^{1} \rightarrow \mathrm{V}^{0}[1], \mathrm{N}^{2}$

We have well-formed Gapping sentences like 24 corresponding to sentences like 42. 44a induces the gapped conjunct of 24 and the resulting structure is $44 \mathrm{~b}$ :

(44) a. S[ELL, [CONJ and]] $\rightarrow$ and, $\mathrm{N}^{2}[\mathrm{FOC}], \mathrm{N}^{2}[\mathrm{FOC}]$

b.

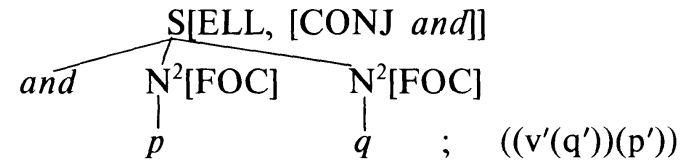

Notice that there is no verb in $44 \mathrm{a}$, but that its semantic translation contains a denotation of $\mathrm{v}$, which is identical to the verb in the nongapped conjunct 41 . The structure of 24 looks like 45a:

(45)

a.

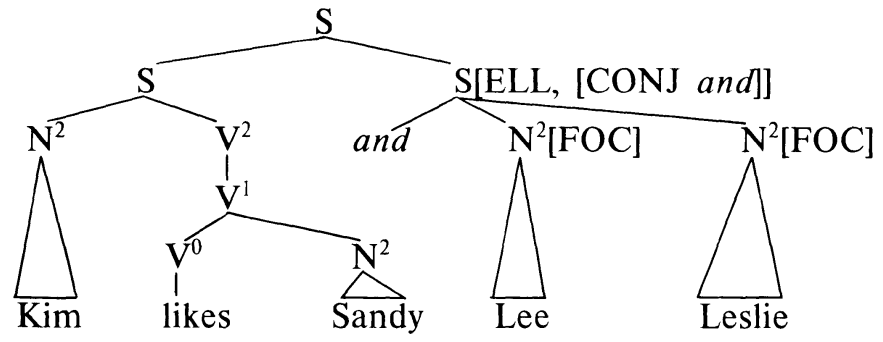

b. and'(like'(Sandy*)(Kim*), like'(Leslie*)(Lee*)

The relation between structures 41 and 45 can be stated like this: if the rules responsible for structure 41 are in the grammar, then rule 44a is also in the grammar. One can define the grammar which admits structure 45 in an inductive way as in 46.

(46)

$$
\begin{aligned}
& \left\{\begin{array}{l}
\mathrm{S} \rightarrow \mathrm{N}^{2}, \mathrm{~V}^{2}[\text { TNS t }] \\
\mathrm{V}^{2} \rightarrow \mathrm{V}^{1}[\text { TNS } \mathrm{t},[1]] \\
\mathrm{V}^{1}[\mathrm{TNS} \mathrm{t},[1]] \rightarrow \mathrm{V}^{0}\left[\text { TNS t, [1]], } \mathrm{N}^{2}\right.
\end{array}\right\} \\
& \Longrightarrow\left\{\begin{array}{l}
\mathrm{S} \rightarrow \mathrm{S}[\mathrm{TNS} \mathrm{t}], \mathrm{S}[\mathrm{ELL},[\mathrm{CONJ} \alpha]] \\
\mathrm{S}[\text { ELL, [CONJ } \alpha]] \rightarrow \alpha, \mathrm{N}^{2}[\mathrm{FOC}], \mathrm{N}^{2}[\mathrm{FOC}]
\end{array}\right.
\end{aligned}
$$

Next, let us consider examples with verbs like be, seem, consider and become. These verbs are introduced by the following ID rules:

(47)
a. $\mathrm{V}^{1} \rightarrow \mathrm{V}^{0}[3], \mathrm{X}^{2}[\mathrm{PRD}]$
(V[3]: be $)$
b. $\mathrm{V}^{1} \rightarrow \mathrm{V}^{0}[4], \mathrm{X}^{2}[\mathrm{PRD},+\mathrm{N}]$
(V[4]: become)
c. $\mathrm{V}^{1} \rightarrow \mathrm{V}^{0}[5], \mathrm{N}^{2}, \mathrm{X}^{2}$ [PRD, -PRP]
(V[5]: consider)
d. $\mathrm{V}^{1} \rightarrow \mathrm{V}^{0}[6],\left(\mathrm{P}^{2}[t o]\right), \mathrm{V}^{2}[\mathrm{INF}]$
(V[6]: seem)

These account for 30 and 31 . 
As SGWW suggest, given 47, all well-formed conjuncts in 30 and 31 can be defined in the same way as in 46 .

Let us turn to more complex cases. The Binary Coordination Schema (BCS) 7, the Iterative Coordination Schema (ICS) 8 and the LP statement for conjunctions will take care of the distribution of conjunctions in Gapping sentences. Furthermore, the following LP (Linear Precedence) statement is operative:

(48) LP statement for the feature ELL

$$
[-\mathrm{ELL}]<[\mathrm{ELL}]
$$

LP statement 48 says that categories specified $<$ ELL, $+>$ cannot precede categories specified $<$ ELL, $\rightarrow>$.

The interaction of 7, 9, 46 and other independently motivated ID rules admit, for example, the following Binary Gapping sentences:

(49) a. John likes coffee or Bill tea.

b. Either John likes coffee or Bill tea.

c. Neither John likes coffee nor Bill tea.

Let us turn to the case of Gapping containing more than one gapped conjunct (Iterative Gapping sentences). It is possible to add gapped conjuncts to Gapping sentences. Gapping process may occur iteratively. For example, sentences like 50 are grammatical:

(50) a. John likes Mary, Bill Jane, and Harry Susan.

b. Alice ordered pork chops, Ben liver and Sylvia lasagna. Iterative Gapping sentences like 50a-b can be defined in the same way as Binary Gapping sentences. If some simple $\mathrm{S}$ is admitted by the grammar, then $S+$ gapped $S$ is also admitted by the grammar, and further $\mathrm{S}+$ gapped $\mathrm{S}+$ gapped $\mathrm{S}+\ldots$ is also admitted by the grammar. Appropriate distribution of conjuncts is attained through interaction of independently established ID rules 7, 8, 9 and 48 .

Consider 51.

(51) a. John likes Mary, Bill Jane, Harry likes Susan.

b. Alice ordered pork chops, Ben liver, and Sylvia ordered lasagna.

McCawley (1981: 78-79) cites the following sentences (judgments are his):

(52) a. Alice ordered pork chops, Ben liver, and Sylvia lasagna.

b. ?Alice ordered pork chops, Ben liver, and Sylvia ordered lasagna.

c. ?Alice ordered pork chops, Ben ordered liver, and Sylvia lasagna. 
McCawley's judgments suggest that $52 \mathrm{~b}-\mathrm{c}$ are not necessarily ungrammatical. The ellipsis causes no processing problem, since they lack no more elements than Iterative Gapping sentences like 52a. Gapping occurs iteratively to all conjuncts but the first; if not, the acceptability of the resulting sentences decreases.

Consider the following sentences:

(53) a. I know that John likes coffee and Bill tea.

b. I persuaded Linda (that) Alice ordered pork chops, Ben liver, and Sylvia lasagna.

c. Tom conceded to the teachers that John loved Mary and Bill Sue.

Notice that Gapping is defined on S's, and resulting Gapping sentences are also S's. The interaction of the following ID rules and the rules 79 and 48 generates $53:{ }^{11}$

(54) $\mathrm{V}^{1} \rightarrow \mathrm{V}^{0}$ [7], S[FIN]

(55) $\mathrm{V}^{1} \rightarrow \mathrm{V}^{0}[8],\left(\mathrm{P}^{2}[t o]\right), \mathrm{S}[\mathrm{FIN}]$

(V[7]: know, believe, ...)

(V[8]: concede, admit, ...)

3.2. Cases Involving Adverbial Features. Let us consider Gapping sentences containing elements which are optional in the sense that they do not subcategorize the verbs. The following are examples:

(56) a. John cooked the rice quickly, and Mary the beans slowly.

b. Peter talked to his boss on Tuesday, and Betsy to her supervisor on Wednesday.

Following SGWW's proposal, let us assume three adverbial features MANNER, TEMP, and PLACE. To illustrate:

(57) a. $\mathrm{X}^{2}[\mathrm{MANNER}]: \operatorname{Adv}^{2}[\mathrm{MANNER}]$ : slowly, rapidly, ... : $\mathrm{P}^{2}[\mathrm{MANNER}] \quad$ : with care, ...

b. $\mathrm{X}^{2}[$ TEMP $] \quad$ : $\mathrm{P}^{2}$ [TEMP] $\quad$ : on Tuesday, in $1985, \ldots$ : $\mathrm{N}^{2}[\mathrm{TEMP}] \quad$ : yesterday, ...

c: $\mathrm{X}^{2}[\mathrm{PLACE}] \quad$ : $\operatorname{Adv}^{2}[\mathrm{PLACE}] \quad$ : outside, abroad, ... : $\mathrm{P}^{2}[\mathrm{PLACE}] \quad$ : in the garden, ...

Generally there seems to be a correspondence between the two conjuncts of Gapping sentences including optional elements.

We might propose the following conditions on Gapping.

(58) General condition on Gapping I

"For a discussion of complementizers within a framework of GPSG, see Weeda 1981. 
The number of $X^{2}$ elements (called TERMS) in the nongapped conjunct is equal to the number of $\mathrm{X}^{2}$ ELEMENTs in the gapped conjunct.

(59) General condition on Gapping II $^{12}$

One necessary condition on the well-formedness of Gapping is that each TERM in the gapped conjunct forms a TRUE PAIR with the corresponding TERM in the nongapped conjunct. With respect to optional elements, if a TERM in the nongapped conjunct and the corresponding TERM in the gapped conjunct bear the same feature, then they form a TRUE PAIR. These two conditions are difficult to incorporate in syntax. They may be conditions in pragmatics.

$56 \mathrm{a}$ is admitted by the grammar in the following way. First, the following structure is independently motivated:

(60)

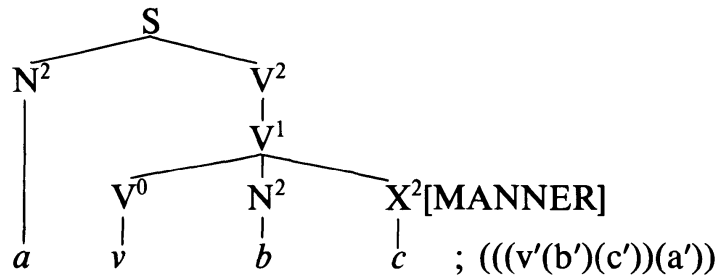

The gapped structure corresponding to 60 is 61 , and is admitted by the grammar.

(61)

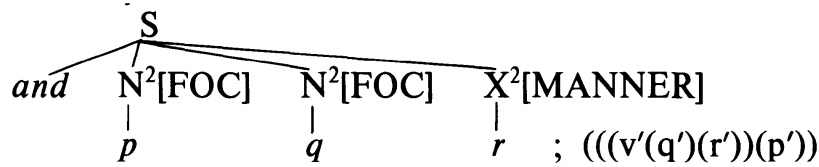

If the grammar contains the rules responsible for $60-61$, then conjoined sentence $60+61$ is admitted by the grammar.

One can extend the relationship between 60 and 61 in the following way:

12 Consider the following sentences (judgments are Jackendoff's (1971)):

i) *John quickly cooked the rice and Maggy slowly the beans.

ii) *John quickly cooked the rice and Maggy quickly the beans.

iii) John quickly cooked the rice and Maggy $\phi j d j / \phi \mid k \psi y \phi \phi \phi k \phi \phi d$ the beans. A manner adverb identical to the manner adverb in the nongapped conjunct cannot appear in the gapped conjunct, as illustrated in the contrast between ii and iii. If they are not identical, Gapping cannot occur, as illustrated in the ungrammaticality of $i$. It may be that a manner adverb in the preverbal position becomes a TERM together with the verb which follows it. But one of my informants observed that the contrast between them are not so clear-cut. 
(62)

a.

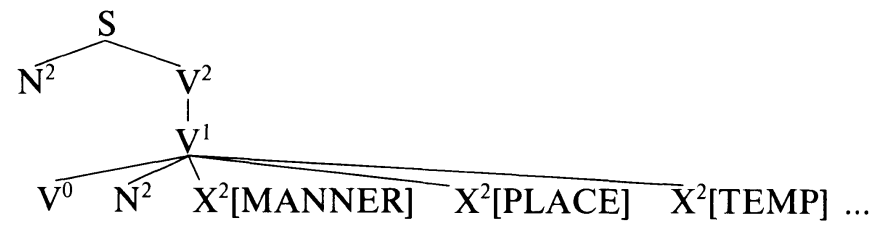

b.

S[ELL, [CONJ $\alpha]]$

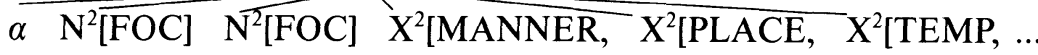
[FOC] $\quad[\mathrm{FOC}]] \quad[\mathrm{FOC}]]$

Notice that the order of remnants may vary. ${ }^{13}$

3.3. Complex Examples of Gapping. Neijt (1979: 35-36) gives the following sentences:

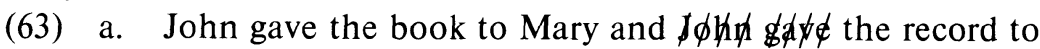
Sue, or $\ \phi h \phi d q y \phi$ the book to Sue and $\$ \phi$ hh $\$ d y \phi$ the record to Mary.

b. John invited Sue and Max inyitted Mary, or Max indiled

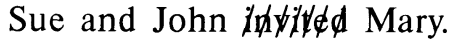

(64) a. *John gave the book to Mary and he handed the record to Sue, or he $\$ d y \notin$ the book to Sue and he happdqd the - record to Mary.

b. *John saw Sue and Max invited Mary, or Max $\$ d w$ Sue and John Indyitted Mary.

The sentences in 64 cause no problem to our analysis, since the identity of the verb is guaranteed by 44 . But the sentences in 63 are not admitted by our analysis. Compare them with the following sentences:

(65) a. John gave the book to Mary and the record to Sue, or John gave the book to Sue and the record to Mary.

b. John invited Sue and Max Mary, or Max invited Sue and John Mary.

13 Sentences like 25 in which an indefinite number of infinitives follow the gapped main verb have not been considered so far. GPSG assumes two kinds of conditions on syntactic features, i.e. Feature Cooccurrence Restrictions (FCR's) and Feature Value Defaults (FVD's). FCR's are absolute conditions on possible syntactic categories (see GKPS in press for further discussion). If, following Gazdar, Pullum, and Sag 1982, features FIN = finite, INF = infinitive, and BSE = base form are adopted, then a FCR like [ELL] $\supset[-B S E]$ would block strings like try to go to Rome or go to Rome as remnants. FCR [ELL] [-BSE] says that syntactic categories which are specified $<$ ELL, $+>$ cannot be specified $<\mathrm{BSE},+\rangle$. In other words, categories like $\{<\mathrm{ELL},+\rangle,\langle\mathrm{BSE},+\rangle, \ldots\}$ are not legal categories. 
65 are generated because simple Gapping sentences are S's and therefore can be embedded or coordinated as such. Iterative application of embedding or coordination are permitted, but resulting sentences will soon become hard to process. There is an obvious relationship between 63 and 65 , but at present it is not clear how our analysis can be modified to admit sentences like 63 .

\section{Conclusion}

As for the UNITARY NOTION OF COORDINATION discussed in Section 1.3, Gapping shares this notion of coordination indirectly in the proposed analysis. Gapping is different from regular coordination in that gapped conjuncts are dependent on the preceding nongapped conjuncts. This dependency relation allows gapped conjuncts to be elliptical. Therefore, I have proposed to define Gapping as dependent on nongapped conjuncts in its generation.

Diagrammatically, the grammar of English sentences, excluding elliptical sentences, is as shown in Figure 3.

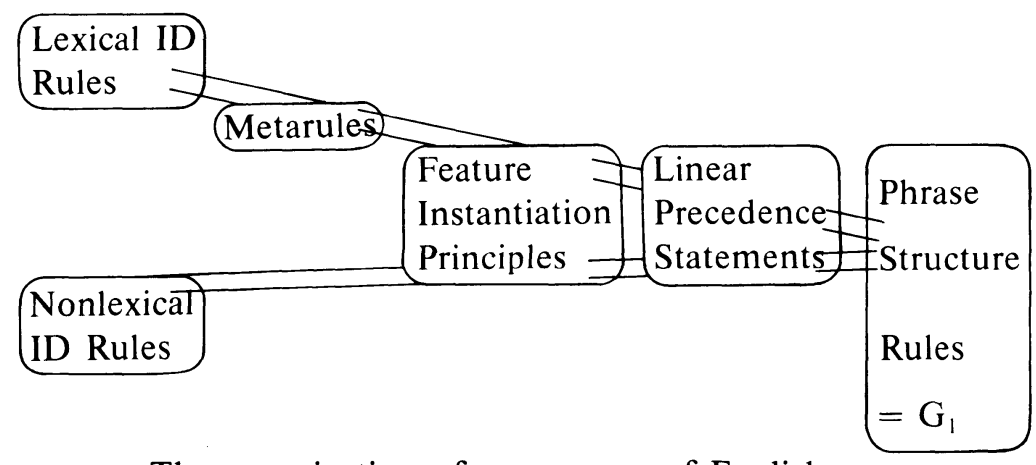

The organization of a grammar of English sentences, excluding elliptical sentences

Figure 3.

$\mathrm{G}_{1}$ is a set of rules which directly generate actual sentences. My proposal for the generation of Gapping sentences is that if some simple $S$ is in the grammar, i.e. admitted by the rules in $G_{1}$, then the grammar also contains the rules responsible for the corresponding gapped conjuncts. Rules for gapped conjuncts are defined in an inductive way.

$G_{1}$ contains a set of rules $P_{1}$, which give rise to simple $S$ 's and apart from $G_{1}$ there is also a set of rules $Q_{1}$ for gapped conjuncts which are 
specified for the feature ELL + . These two sets of rules give rise to Gapping sentences by interacting with the rules which are independently motivated for regular coordination, namely, Binary Coordination Schema (BCS), Iterative Coordination Schema (ICS), the LP statement for conjunctions and so on. The relation between these two sets of rules is as shown in Figure 4.
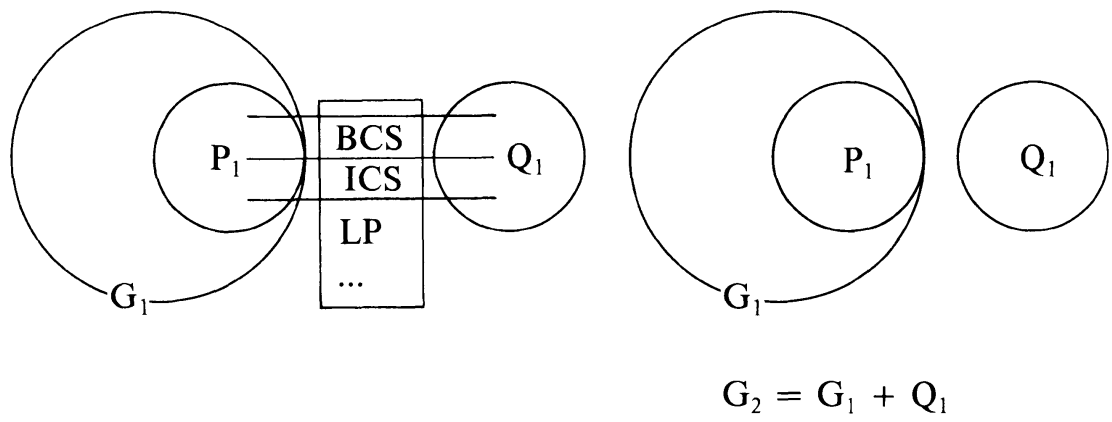

The relationship between the rules for nongapped conjuncts and the rules for gapped conjuncts

Figure 4.

A problem immediately arises. GPSG assumes that all the ID rules exist on the left-hand side in Figure 3. Moreover, the question of how the features ELL and FOC are assigned is not solved but simply assumed in the proposed analysis. Two alternatives suggest themselves. First, it is conceivable to move the rules in $\mathrm{Q}_{1}$ to the left-hand side in Figure 3. and assign feature ELL and FOC differently from other features. The rationale for this may be that ID rules for gapped conjuncts can be treated in the same way as other ID rules are, and that the feature ELL and FOC are different from other features. But this alternative requires some mechanism for insuring a correspondence between the nongapped conjunct and the gapped conjunct(s) and the identity of the verb in question. The other alternative is to postulate a separate component where elliptical phrases are handled on the right-hand side in Figure 3. But this alternative raises the immediate question of whether or not this component unduly increases the expressive power of the generated grammar.

The analysis of Gapping given in Section 3 is along the latter alternative. The mechanism of generation of Gapping sentences is as shown in Figure 5. 


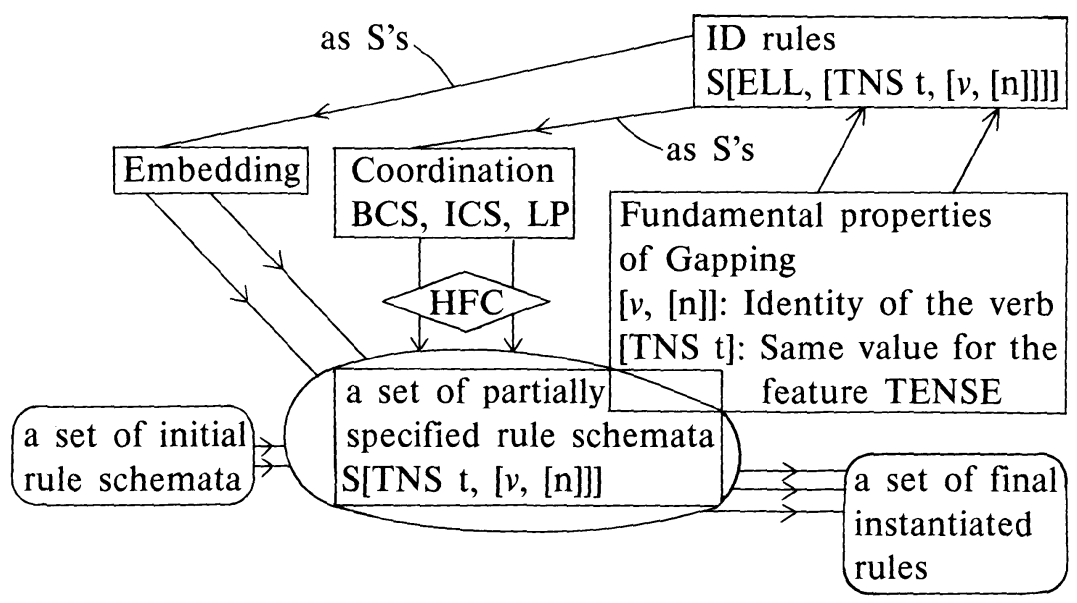

Figure 5.

\section{REFERENCES}

Сномsкy, Noam. 1970. Remarks on nominalization. Readings in English transformational grammar, ed. by Roderic A. Jacobs and Peter S. Rosenbaum, 184-221. Waltham, Mass: Ginn and Company.

Farkas, Donka, Daniel P. Flickinger, Gerald Gazdar, William A. Ladusaw, Almerindo Ojeda, Jessie Pinkham, Geoffrey K. Pullum, and Peter Sells. 1983. Some revisions to the theory of features and feature instantiation. Proceedings of ICOT Workshop on Non-Transformational Grammars. 11-3. Tokyo: Institute for New Generation Computer Technology.

Flickinger, Daniel. 1983. Lexical heads and phrasal gaps. Michael Barlow, Daniel Flickinger, and Michael Wescoat eds. Proceedings of the Second West Coast Conference on Formal Linguistics. 89-101. Stanford Linguistics Association, Department of Linguistics, Stanford University.

Gazdar, Gerald. 1981. Unbounded dependencies and coordinate structure, LI 12.155-84.

1982. Phrase structure grammar. The nature of syntactic representation, ed. by Pauline Jacobson and Geoffrey K. Pullum, 131-86. Dordrecht, Holland: D. Reidel.

, Ewan Klein, and Geoffrey K. Pullum (eds.) 1983. Order, concord and constituency. Dordrecht, Holland: Foris.

, Ewan Klein, Geoffrey K. Pullum, and Ivan A. Sag. 1982. Coordinate structure and unbounded dependencies. Developments in Generalized 
Phrase Structure Grammar, ed. by Barlow, Michael, Daniel P. Flickinger and Ivan A. Sag, 38-68. Bloomington, Indiana: Indiana University Linguistics Club.

, Ewan Klein, Geoffrey K. Pullum, and Ivan A. Sag. In press. Generalized phrase structure grammar. Cambridge, Mass: Harvard University Press/ Oxford: Basil Blackwell.

and Geoffrey K. Pullum. 1981. Subcategorization, constituent order, and the notion "head". The scope of lexical rules, ed. by M. Moortgat, H.v.d. Hulst, and T. Hoekstra, 107-23. Dordrecht, Holland: Foris.

and Geoffrey K. Pullum. 1982. Generalized phrase structure grammar: a theoretical synopsis. Bloomington, Indiana: Indiana University Linguistics Club.

, Geoffrey K. Pullum, and Ivan A. Sag. 1982. Auxiliaries and related phenomena in a restrictive theory of grammar. $\mathrm{Lg} 58.591-638$.

Geoffrey K. Pullum, Ivan A. SAg, and Thomas Wasow. 1982. Coordination and transformational grammar. LI 13.663-76.

GunjI, TAKaO. 1983. Introduction to linguistics for computer scientists, (in Japanese). Tokyo: Information-Technology Promotion Agency.

. In preparation. A phrase structural analysis of the Japanese language.

HANKAMER, JORGE. 1973. Unacceptable ambiguity. LI 4.17-68.

1979. Deletion in coordinate structures. New York: Garland Publishing.

Hudson, Richard A. 1982. Incomplete conjuncts. LI 13.547-50.

JACKENDOFF, RAY S. 1971. Gapping and related rules. LI 2.21-35.

KaPLAN, JoHn. 1984. Obligatory too in English. Lg 60.510-18.

Klein, Ewan and Ivan A. SAG. 1982. Sematic type and control. Developments in generalized phrase structure grammar, ed. by Michael Barlow, Daniel P. Flickinger, and Ivan A. Sag, 1-25. Bloomington, Indiana: Indiana University Linguistics Club.

MCCAWLEY, JAmES D. 1981. Everything that linguists have always wanted to know about logic* *but were ashamed to ask. Chicago, Ill: University of Chicago Press.

Montague, Richard. 1974. Formal philosophy: selected papers of Richard Montague, ed. by R. H. Thomason. New Haven, Conn: Yale University Press.

Neijt, Anneke. 1979. Gapping. Dordrecht, Holland: Foris.

Pullum, Geoffrey K. and Gerald Gazdar. 1982. Natural languages and contextfree languages. Linguistics and Philosophy 4.741-88.

Sag, Ivan A., Gerald Gazdar, Thomas Wasow, and Steven Weisler. 1984. Coordination and how to distinguish categories. Report No. CSLI-84-3. Center for the Study of Language and Information. Stanford University.

Terazu, Noriko. 1975. Coordinate deletion, gapping, and right node raising. Studies in English Linguistics 3.19-65. Tokyo: Asahi Press.

Weeda, Donald. 1981. Tenseless that-clauses in generalized phrase structure grammar. Roberta A. Hendrick, Carrie S. Masek, and Mary Frances Miller eds. Proceedings of the seventeenth regional meeting of Chicago Linguistic Society. 404-10. Chicago Linguistic Society, The University of Chicago.

Williams, Edwin. 1978. Across-the-Board rule application. LI 9.31-43. 